\title{
Polyherbal formulation Anoac-H suppresses the expression of RANTES and VEGF for the management of bleeding hemorrhoids and fistula
}

\author{
ASHWIN PORWAL ${ }^{1}$, GOPAL C. KUNDU ${ }^{2,3}$, GAJANAN BHAGWAT ${ }^{4}$ and RAMESH BUTTI ${ }^{2,4}$ \\ ${ }^{1}$ Healing Hands Clinic, Pune, Maharashtra 411001; ${ }^{2}$ Laboratory of Tumor Biology, \\ Angiogenesis and Nanomedicine Research, National Centre for Cell Science, Pune, Maharashtra 411007; \\ ${ }^{3}$ School of Biotechnology and Kalinga Institute of Medical Sciences, KIIT Deemed to be University, \\ Institute of Eminence, Bhubaneswar, Odisha 751024; \\ ${ }^{4}$ Healing Hands \& Herbs (R\&D Center), Pune, Maharashtra 411002, India
}

Received April 4, 2021; Accepted July 21, 2021

DOI: $10.3892 / \mathrm{mmr} .2021 .12376$

\begin{abstract}
Hemorrhoids and fistula are considered the most common anorectal conditions in the general population. These conditions affect the quality of a patient's life by causing pain and bleeding during defecation or even in the resting state. Lower grades of hemorrhoids may be controlled by traditional measures. However, surgery is an effective treatment option in recurrent-lower and higher-grade hemorrhoids. Surgical procedures are associated with various complications, including pain and delayed wound healing. Recurrence of hemorrhoids is also a major concern in the post-operative period. An anal fistula is the connection between the anus and the skin and causes severe pain, swelling, as well as blood and pus discharge. Fistula has serious social and economic consequences. Hence, it is important to understand the pathophysiology and molecular pathology of hemorrhoids and fistula, to identify the molecular targets and to develop pharmacological-interventions. In a previous study by our group, the polyherbal formulation Anoac-H was developed for the treatment of different stages of hemorrhoids and fistula, and it was demonstrated that Anoac-H is an effective formulation for treating hemorrhoids. However, the molecular mode of action of Anoac-H on hemorrhoids and fistula had remained elusive. In the present study, it was determined that this formulation reduces the migration of mesenchymal (fibroblasts) and immune (RAW 264.7) cells without affecting their viability. It was also observed that Anoac-H suppresses the expression
\end{abstract}

Correspondence to: Dr Ashwin Porwal, Healing Hands Clinic, 4th Floor, Millennium Star Extension Building, Building Number-B, Dhole Patil Road, Pune, Maharashtra 411001, India

E-mail: drashwinporwal@healinghandsclinic.co.in

Key words: Anoac-H, PiloTab, hemorrhoids, wound migration, cell viability, regulated upon activation, normal $\mathrm{T}$ cell expressed and presumably secreted, VEGF of regulated upon activation, normal $\mathrm{T}$ cell expressed and presumably secreted (RANTES) and VEGF in fibroblasts and macrophages. Inflammation and elevated expression of RANTES and VEGF were observed in hemorrhoids and fistula. However, inflammation, as well as the expression of RANTES and VEGF, were significantly reduced in treated human hemorrhoid and fistula tissues as compared to untreated ones, confirming the in vitro results.

\section{Introduction}

Anorectal pathologies such as hemorrhoids and fistula are common conditions affecting normal functions of anus and rectum (1). Hemorrhoids are symptomatic enlargement and distal displacement of vascular anal cushions. It is estimated that $75 \%$ of the world population experiences enlarged hemorrhoids at a certain point of time in their lives (2). The incidence of hemorrhoids is equally in males and females, with the highest occurrence rate between 45 and 65 years of age $(2,3)$. A total of $40 \%$ of patients with hemorrhoids exhibit no symptoms (3). However, pain, itching, swelling, anal discomfort and rectal bleeding are observed in symptomatic patients (4). Multiple factors contribute to the development of hemorrhoids that include prolonged straining, diarrhea, constipation, overweight, obesity, pregnancy and old age (4-6). Previous studies indicated that severe inflammatory reaction takes place in the vascular wall and the surrounding connective tissue of the hemorrhoids and it gradually leads to mucosal ulceration, ischemia and thrombosis (7). In this area, tissue damage induces an inflammatory response, which manifests as infiltration of T-lymphocytes, macrophages, neutrophils, monocytes, mast cells and dendritic cells (8). These cells secrete both antiand proinflammatory cytokines. However, due to excessive production of proinflammatory cytokines, the proinflammatory response gradually overtakes the anti-inflammatory response $(8,9)$. IL-1 $\beta$, IL-6, regulated upon activation, normal $\mathrm{T}$ cell expressed and presumably secreted (RANTES), VEGF and TNF- $\alpha$ are considered proinflammatory cytokines which have a critical role in human health and disease $(10,11)$. 
RANTES is a C-C motif chemokine ligand 5, has a critical role in inflammation by mobilizing different types of immune cells $(12,13)$. The proangiogenic factor VEGF is highly present at these sites and involved in the advancement of hemorrhoidal disease by regulating increased vascular density (7).

An anal fistula is considered an epithelialized tract or a connection between the anal canal and the perianal skin. Classic anal fistulas are the outcome of a perineal infection and abscess formation (14). Fistulas are also associated with inflammatory bowel disease, Crohn's disease, radiation, malignancy, chronic diarrhea or pre-existing incontinence (15). Expression of RANTES and VEGF is observed in Crohn's disease-related perianal fistula and arteriovenous fistulas $(16,17)$. Fistulectomy is a common operative procedure for the treatment of fistula (18). These procedures are associated with postoperative complications, anal incontinence and recurrence (19). Hemorrhoids and fistulas are associated with inflammation. Different types of cells, such as macrophages, neutrophils, and fibroblasts, are present in the inflammatory environment of hemorrhoids and fistulas $(8,15,20-22)$. Natural remedies for hemorrhoids and fistula, including laxatives, analgesics and anti-inflammatory agents, have drawn much attention recently due to their relatively higher effectiveness, low cost and lower invasiveness $(23,24)$. A study by our group, for the first time, reported that Anoac-H, a polyherbal formulation, exhibits safety and efficacy in treating bleeding hemorrhoids (25). The present study aimed to investigate the mechanism of action of Anoac-H in the treatment of bleeding hemorrhoids and fistula by studying its effect on the expression of proinflammatory and proangiogenic factors.

\section{Materials and methods}

Cell culture. Fibroblasts and macrophages were used as a model for inflammatory cells present in hemorrhoids and fistulas. The human foreskin fibroblast cell line BJ and the mouse monocyte/macrophage-like cell line RAW 264.7 were purchased from the American Type Culture Collection. BJ and RAW 264.7 cells were cultured in MEM(E) (HiMedia Laboratories LLC) and RPMI (Gibco; Thermo Fisher Scientific, Inc.) media respectively, supplemented with $10 \%$ fetal bovine serum (Gibco; Thermo Fisher Scientific, Inc.) and 100 units of penicillin and $100 \mu \mathrm{g} / \mathrm{ml}$ streptomycin at $37^{\circ} \mathrm{C}$ in a humidified atmosphere with $5 \% \mathrm{CO}_{2}$. All treatments were performed in complete medium.

Drug preparation. Anoac-H/PiloTab (Healing Hands \& Herbs, https://healinghandsandherbs.in/) is an ayurvedic polyherbal formulation in the form of a tablet. Anoac-H tablet consists of Lajjalu (Mimosa pudica), Dugdhika (Euphorbia hirta), Nagkesar (Messua ferrea) and Daruharidra (Berberis aristata) extracts, as described in a previous study (25). For the treatment of the cells, the tablet was minced and dissolved in sterile water to prepare a stock of $100 \mathrm{mg} / \mathrm{ml}$.

Cell viability assay. Cell viability was studied using the MTT assay, as per the previously described protocol (26). In brief, BJ/RAW 264.7 cells $\left(1 \times 10^{4}\right)$ were seeded into 96-well flat-bottom microplates and treated with vehicle control (equal volume of water) or Anoac-H at different concentrations
$(25-500 \mu \mathrm{g} / \mathrm{ml})$ for $24 \mathrm{~h}$. To each well, MTT $(0.5 \mathrm{mg} / \mathrm{ml})$ solution was added, followed by incubation for $4 \mathrm{~h}$ at $37^{\circ} \mathrm{C}$. Isopropanol was then added to dissolve the formazan crystals and the optical density of the formazan solution was measured at $570 \mathrm{~nm}$ using an automated microplate reader $(\mathrm{EPOCH} 2$; Agilent Technologies, Inc.). All experiments were performed in biological triplicates.

Migration assay. Cell migration was studied by a wound closure assay as per the standard procedure described previously $(27,28)$. In brief, BJ/RAW $264.7\left(2 \times 10^{5}\right)$ cells were seeded in 12-well plates and allowed to attain confluency. When cells achieved confluency, a line-shaped scratch was made in the monolayers with a sterile $200-\mu$ l pipette tip. Cells were either treated with vehicle control (equal volume of water) or Anoac- $\mathrm{H}$ at different concentrations (100 and $250 \mu \mathrm{g} / \mathrm{ml})$. Images were captured at 0 and 12/16 h using a Nikon phase-contrast microscope (Nikon Corp.). The area of wound closure was analyzed by Image-Pro Plus 6.0 software (National Institutes of Health).

Western blot analysis. Western blot was performed to examine the expression of proangiogenic and proinflammatory proteins in control or treated cells according to a standard protocol (29). In brief, cells were harvested and lysed using RIPA lysis buffer. Protein concentration of cell lysates was determined using a Bradford assay. Equal amounts of total protein (30 $\mu \mathrm{g})$ were resolved by SDS-PAGE (10 or $12.5 \%$ gels). The resolved proteins were then transferred onto a PVDF membrane (Bio-Rad Laboratories, Inc.) and processed for analysis. The membrane was incubated with antibodies to RANTES (Santa Cruz Biotechnology, Inc., cat. no. sc-1410, 1:1,000 dilution), VEGF (Santa Cruz Biotechnology, Inc., cat. no. sc-7269, 1:1,000 dilution), IL-1 $\beta$ (Elabscience Biotechnology Inc; cat. no. E-AB-52153, 1:1,000 dilution) overnight at $4^{\circ} \mathrm{C}$, followed by respective horseradish peroxidase (HRP) antibodies (anti-goat HRP, cat. no. sc-2020, anti-rabbit HRP, cat. no. sc-2005, anti-mouse HRP, cat. no. sc-2004, Santa Cruz Biotechnology, Inc., 1:2,000 dilution) for $1 \mathrm{~h}$ at room temperature. $\beta$-actin (Santa Cruz Biotechnology, Inc., cat. no. sc-1615, 1:2,000 dilution) was used as a loading control. All the blots were visualized using Clarity Western ECL (Bio-Rad Laboratories, Inc.) reagent.

Anorectal specimen analysis. The present study was approved by the Institutional Ethics Committee of Healing Hands Clinic (Pune, India). Human hemorrhoid and fistula specimens were collected with the help of a histopathologist from Healing Hands Clinic (Pune, India) with informed consent and the study was performed between February 2020 and December 2020. Paraffin-embedded tissue blocks were cut into 5- $\mu \mathrm{m}$ sections and deposited on poly-L-lysine coated slides. Immunohistochemistry (IHC) was performed using the Super Sensitive Polymer-HRP IHC Detection System (BioGenex) as per the manufacturer's instructions. In brief, the sections were deparaffinized in xylene and subjected to antigen retrieval in a citrate buffer at $90^{\circ} \mathrm{C}$ for $15 \mathrm{~min}$ following rehydration. Sections were covered with peroxide block (3\% hydrogen peroxide in water) and followed by power block (casein and proprietary additives in PBS with $0.09 \%$ sodium azide) of Super Sensitive Polymer-HRP IHC Detection system 

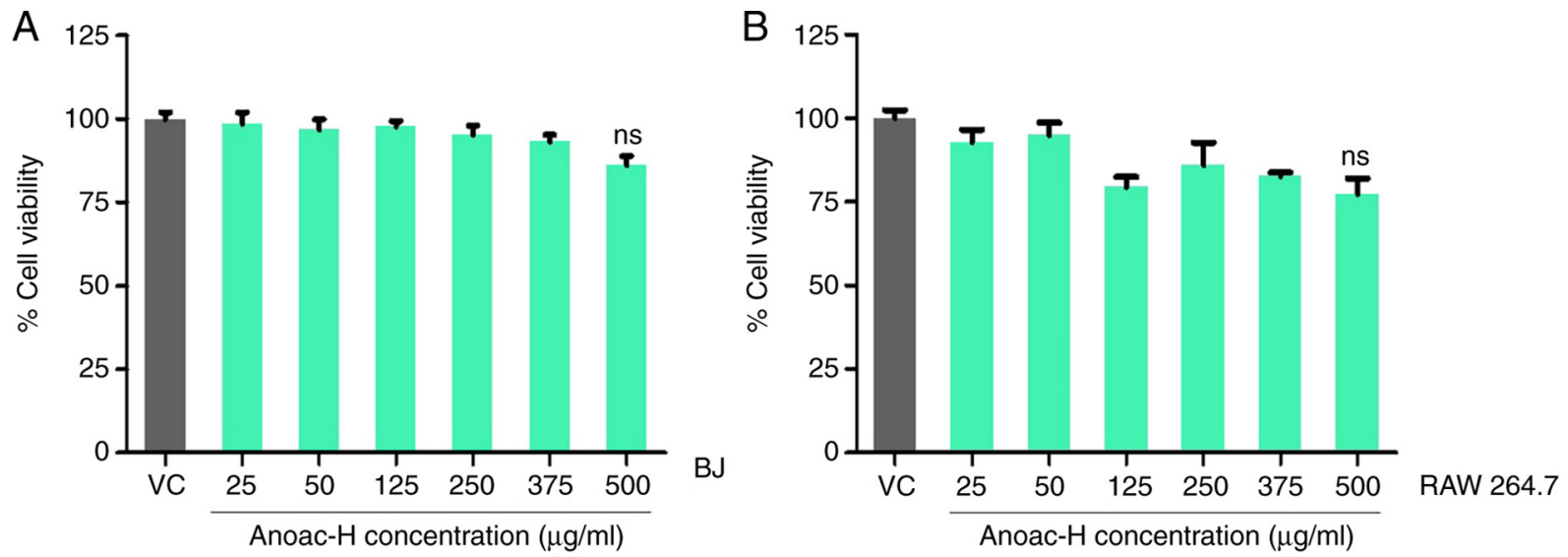

Figure 1. Effect of Anoac-H on cell viability. (A) Fibroblasts were treated with vehicle control or Anoac-H at indicated doses (0-500 $\mu \mathrm{g} / \mathrm{ml})$ for $24 \mathrm{~h}$ and an MTT assay was performed to examine cell viability. (B) RAW 264.7 cells were subjected to treatment with vehicle control or Anoac-H at specified doses $(0-500 \mu \mathrm{g} / \mathrm{ml})$ for $24 \mathrm{~h}$ and an MTT assay was performed to examine cell viability. Values are expressed as the mean \pm standard error of the mean. Kruskal-Wallis test was performed to determine the existence of any statistically significant differences between the vehicle-treated control and the different treatment groups. ns, no significance vs. VC. VC, vehicle control.

(BioGenex Laboratories) to block endogenous peroxidase activity and non-specific binding sites, respectively. Sections were incubated with primary antibodies, such as RANTES (Santa Cruz Biotechnology, Inc., sc-365826, 1:100 dilution) and VEGF (Santa Cruz Biotechnology, Inc., sc-7269, 1:100 dilution), overnight at $4^{\circ} \mathrm{C}$ and subsequently with poly-HRP reagent (from Super Sensitive Polymer-HRP IHC Detection system) for $1 \mathrm{~h}$ at room temperature. Liquid DAB chromogen was added at room temperature for $10 \mathrm{~min}$ and images were captured using a Nikon Eclipse microscope (Nikon Corp.).

Statistical analysis. All experiments were performed in biological triplicates. GraphPad Prism 5 software (GraphPad Software, Inc.) was used for the statistical analysis of the data. Unless indicated otherwise, the results are expressed as the mean \pm standard error of the mean. Statistical significance between two groups was determined by Student's t-test, while one-way ANOVA or the Kruskal-Wallis test was used to determine statistical significance in the case of multiple doses of drug treatments with the Dunn's post-hoc test. $\mathrm{P}<0.05$ was considered to indicate a statistically significant difference.

\section{Results}

Effect of Anoac-H on cell viability. Fibroblasts were treated with the polyherbal formulation Anoac-H to examine its effect on cell viability of the cells. An MTT assay was performed after treatment with vehicle control/Anoac-H at different concentrations $(25-500 \mu \mathrm{g} / \mathrm{ml})$ for $24 \mathrm{~h}$, as per an earlier protocol (26). Kruskal-Wallis was performed to test for statistically significant differences among the groups. The results indicated that Anoac-H did not significantly affect the viability of BJ human fibroblasts as compared to the vehicle control (Fig. 1A). The same experiment was performed on RAW 264.7 mouse macrophage-like cells to observe the effect of Anoac-H on these cells and similarly, the cell viability was not markedly affected (Fig. 1B). From these observations, it may be inferred that Anoac-H does not affect the viability of fibroblasts and macrophages.
Anoac-H suppresses the cell migratory potential. Several cell types infiltrate the site of hemorrhoids and fistula to induce an inflammatory reaction $(8,15,20-22)$. Several cell types, including macrophages and fibroblasts, migrate to the site of inflammation and have a critical role in the inflammatory reaction (20-22). Hence, in the present study, the effect of Anoac-H on the migration of macrophages and fibroblasts was assessed by performing a conventional wound migration assay. Monolayers of fibroblasts and macrophages were wounded and treated with either vehicle control or different concentrations of Anoac-H (100 and $250 \mu \mathrm{g} / \mathrm{ml})$. The effect of Anoac-H on the migration of fibroblasts and macrophages was statistically examined using Student's t-test. The results indicated that Anoac-H significantly suppressed the migration of RAW 264.7 and BJ cells (Fig. 2A-D). Taken together, the present data suggested that Anoac-H exhibits antimigratory effects.

Anoac-H suppresses the expression of proinflammatory and proangiogenic factors. Proinflammatory and angiogenic factors have a critical role in shaping the inflammatory environment during fistula and hemorrhoidal disease progression $(10,16,17,20)$. In the present study, the effect of Anoac-H on the expression of the proinflammatory factors RANTES and IL-1 $\beta$ and the proangiogenic factor VEGF were examined by western blot analysis. The results revealed that the expression of RANTES and VEGF was significantly downregulated upon Anoac-H treatment as compared to the vehicle control in RAW 264.7 cells (Fig. 3A and B). In addition, the expression of another inflammatory cytokine, IL-1 $\beta$, was also reduced by Anoac-H treatment in RAW 264.7 cells (Fig. 3C). A similar experiment was performed using BJ cells. Though expression of these cytokines was less in BJ cells, the results revealed that the expression of RANTES and VEGF was considerably reduced in Anoac-H-treated BJ cells as compared to the vehicle control (Fig. 3D). Collectively, these results demonstrated that Anoac-H significantly suppressed the expression of proinflammatory and proangiogenic factors, RANTES, IL-1 $\beta$ and VEGF, in both macrophages and fibroblasts. 
A
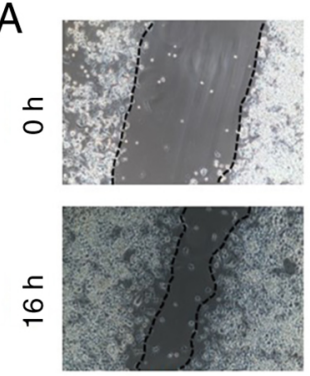

VC
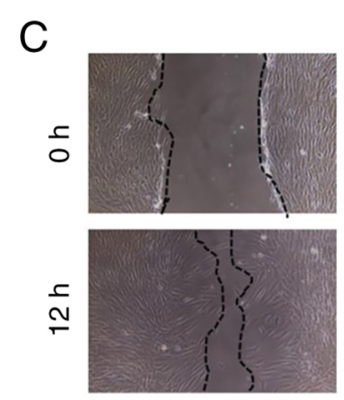

VC
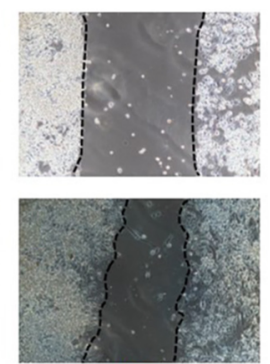

100

Anoac-H $(\mu \mathrm{g} / \mathrm{ml})$
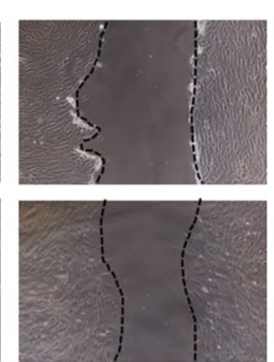

100
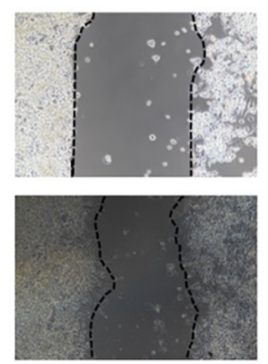

250

RAW 264.7
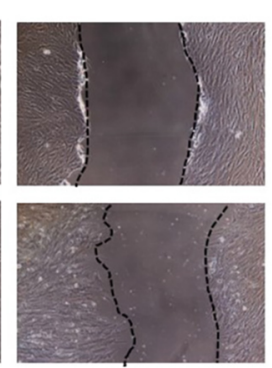

250

BJ

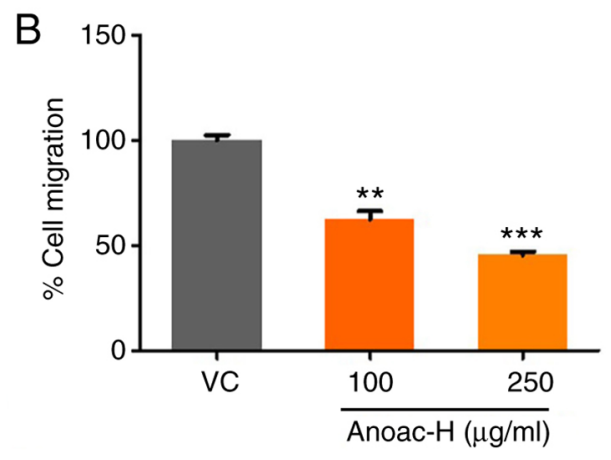

D

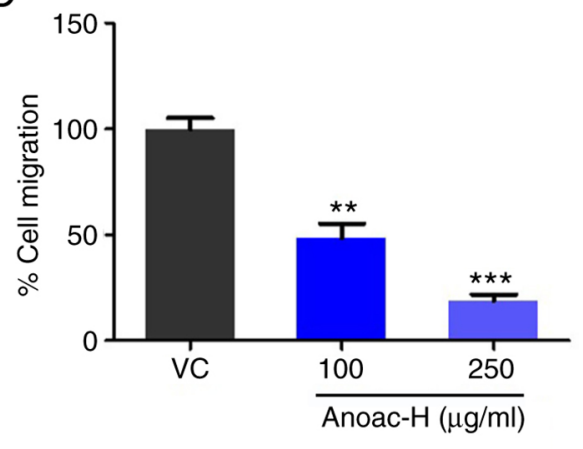

Figure 2. Effect of Anoac-H on cell migration. RAW 264.7 and BJ cells were treated with VC or Anoac-H (0-250 $\mu \mathrm{g} / \mathrm{ml})$ and a wound-healing assay was performed to examine cell migration. Images were acquired at 0 and 12/16 h. (A) Images indicating the effect of Anoac-H on migration of RAW 264.7 cells, were captured using $x 10$ objective. (B) Bar graph representing wound migration assay results from A. (C) Images indicating the effect of Anoac-H on migration of BJ cells, were captured using x10 objective. (D) Bar graph representing wound migration assay results from C. Values are expressed as the mean \pm standard error of the mean. ${ }^{* *} \mathrm{P}<0.01,{ }^{* * *} \mathrm{P}<0.001$ vs. VC. VC, vehicle control.
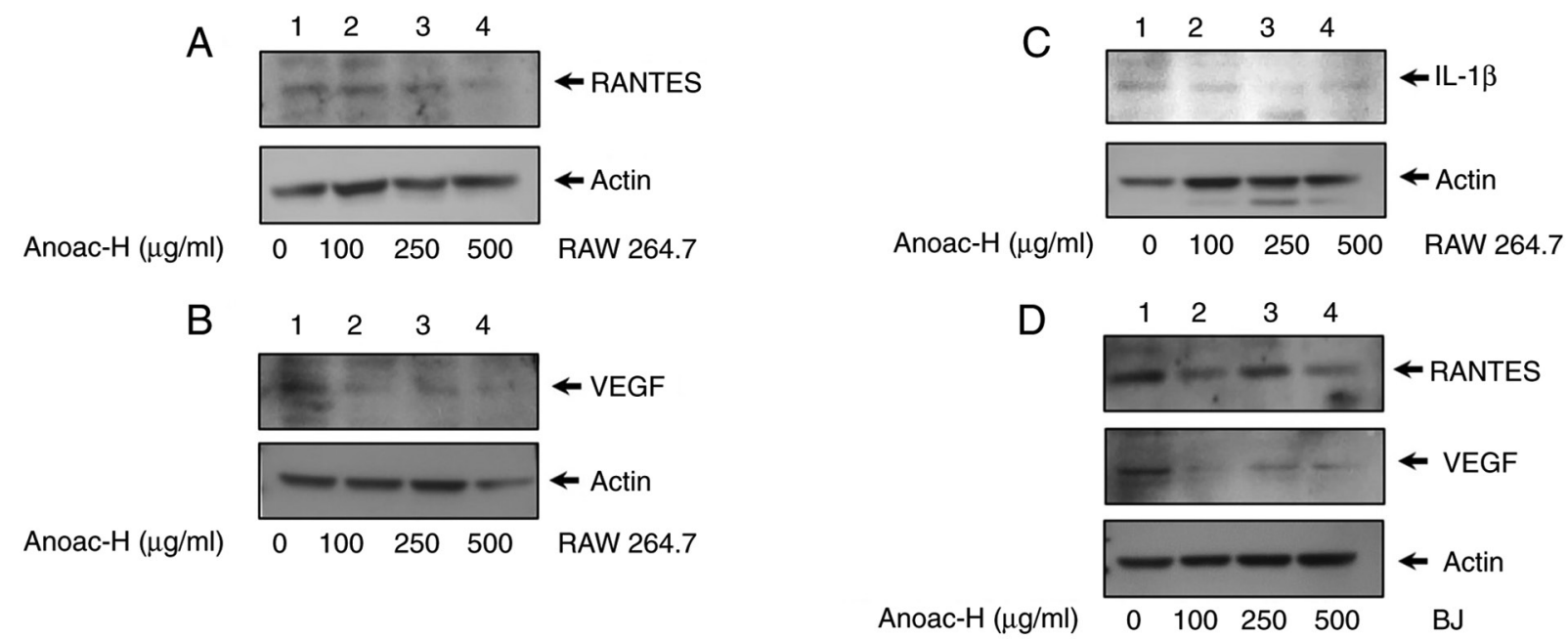

Figure 3. Effect of Anoac-H on the expression of proangiogenic and proinflammatory factors. (A-C) RAW 264.7 cells were treated with vehicle control or Anoac-H $(0-500 \mu \mathrm{g} / \mathrm{ml})$ and the expression of (A) RANTES, (B) VEGF and (C) IL-1 $\beta$ was investigated using western blot analysis. (D) BJ cells were treated with vehicle control or Anoac-H $(0-500 \mu \mathrm{g} / \mathrm{ml})$ and the expression of RANTES and VEGF was analyzed using western blot analysis. RANTES, regulated upon activation, normal $\mathrm{T}$ cell expressed and presumably secreted.

Correlation of RANTES and VEGF expression in patients with anorectal disease. Formalin-fixed, paraffin-embedded human hemorrhoids $(\mathrm{n}=8)$ and fistula specimens $(\mathrm{n}=6)$ were subjected to histological examination by staining with $\mathrm{H} \& \mathrm{E}$. Out of the 14 patients with anorectal disease, 6 patients were treated with Anoac- $\mathrm{H}$, whereas 8 patients were untreated. The age distribution of patients was between 29-65 years (Table SI). A reduction in inflammation was observed in treated specimens as compared to untreated anorectal tissues (Fig. 4A-D). These specimens were also analyzed by IHC to determine the expression of RANTES and VEGF. The IHC data confirmed the expression of RANTES and VEGF in hemorrhoids (Fig. 5A and B). An elevated expression of RANTES and VEGF was also observed in fistula specimens (Fig. 5C and D). Furthermore, it was observed that the expression of RANTES was associated with the expression of VEGF 

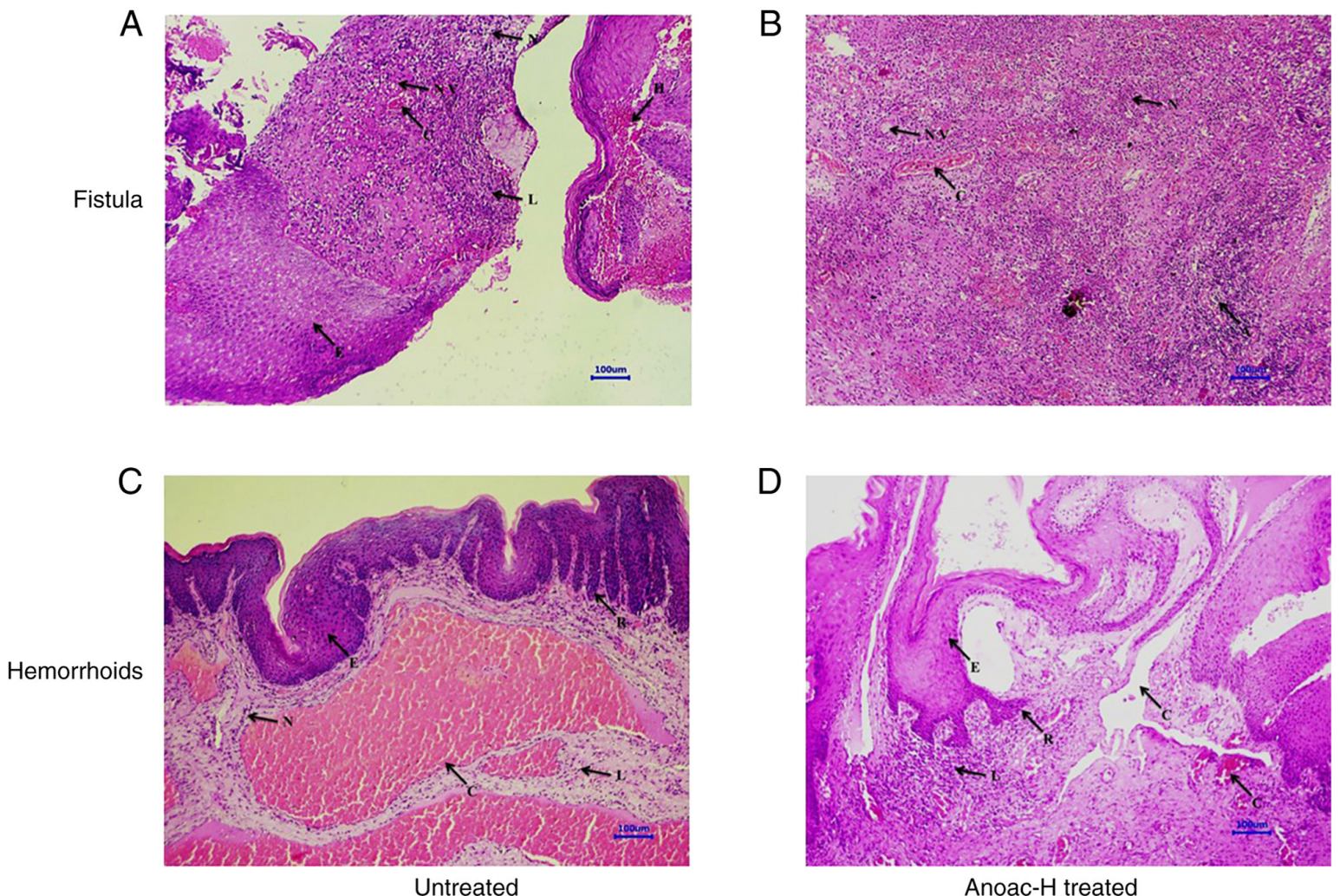

Anoac-H treated

Figure 4. Histological examination of the effect of Anoac-H on tissues of anorectal disease. Untreated or treated hemorrhoids and fistula specimens were stained with H\&E. (A) Untreated fistula, (B) treated fistula, (C) untreated hemorrhoids, (D) treated hemorrhoids. The following features were indicated: $\mathrm{N}$, neutrophil infiltration; NV, neovascularization; L, lymphocyte infiltration; E, epithelial hyperplasia; C, congestion; A, microabscess formation; R, rete ridge formation; and $\mathrm{H}$, hemorrhages (images were captured using x10 objective; scale bar, $100 \mu \mathrm{m}$ ).

in these tissues (Fig. 5A-D). In a previous study by our group, it was determined that Anoac-H helps in the management of hemorrhoids and relieving disease-associated symptoms (25). To determine the association of the expression of RANTES and VEGF with the alleviation of the disease, IHC was performed on Anoac-H-treated hemorrhoids and fistula specimens. Of note, the results indicated that the expression of RANTES and VEGF was drastically reduced in Anoac- $\mathrm{H}$ treated hemorrhoid tissues as compared with untreated patients (Fig. 5E and F). In addition, the results also suggested that the expression of RANTES and VEGF was drastically reduced in Anoac-H treated fistula tissues as compared with untreated patients (Fig. 5G and H). IHC scoring for staining intensity also indicated a significant reduction of RANTES and VEGF expression in the Anoac-H treatment group as compared to untreated anorectal disease tissues (Fig. 6A and B). This is consistent with the present in vitro findings and Anoac- $\mathrm{H}$ inhibiting the expression of RANTES and VEGF may thus reduce inflammation and affect the vasculature to alleviate the disease.

\section{Discussion}

Bleeding hemorrhoids and fistula are common anorectal conditions in humans, but pathologically, they are complex. Patients who present with signs and symptoms of hemorrhoids and fistula require to be carefully evaluated to exclude other masking morbidities $(30,31)$. Hemorrhoids and fistulas are responsible for unrelenting discomfort and cause of distressing pain in patients $(4,15,23,30)$. These anorectal disorders are associated with severe inflammatory reaction. Hemorrhoids are multifactorial diseases and increased vascular density and enlargement of vascular component, as well as disruption of stromal scaffolding are observed in hemorrhoids $(7,32)$. The treatment of hemorrhoids aims at three different aspects. That includes alleviating the immediate symptoms, preventing further exacerbation of the injury and resolving the underlying cause $(4,33)$. Hemorrhoidectomy and fistulectomy are the surgical procedure for removal and management of hemorrhoids and fistula, respectively $(18,34)$. However, these procedures are invasive and associated with postoperative pain, bleeding and fecal incontinence $(7,19)$. Moggia et al $(35)$ have indicated that treatment of hemorrhoid-associated severe bleeding with embolization of superior rectal arteries using coils is safe and effective. Furthermore, relapse of disease is a major limitation of these procedures after a certain postoperative period. Different treatment modalities have been designed to minimize post-operative pain associated with the spasm of the internal sphincter $(36,37)$. Patti et al (38) have reported that the administration of botulinum toxin into the internal anal sphincter after hemorrhoidectomy was successful in decreasing maximum resting pressure, time of wound healing and pain both in the resting state and during defecation without any side effects and complications. Khan et al (39) have achieved efficacy in wound healing and pain relief when they performed trials with a combination of $0.2 \%$ glyceryl trinitrate and 2\% lignocaine ointments after Milligan Morgan hemorrhoidectomy. Another study has also reported that 
A

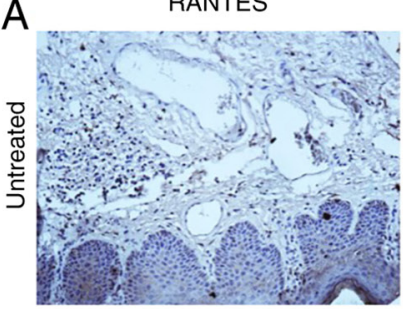

B

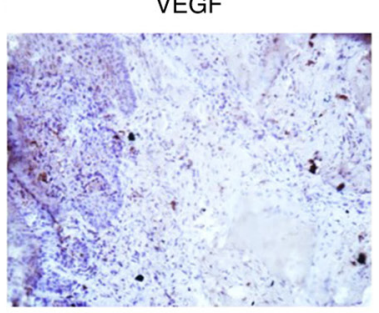

C

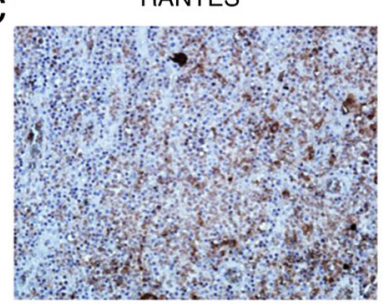

D

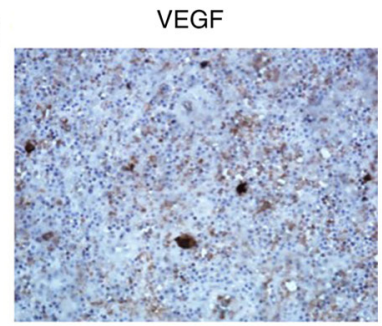

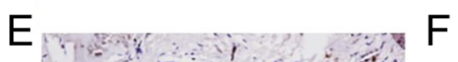

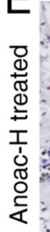

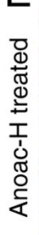

$\mathrm{F}$

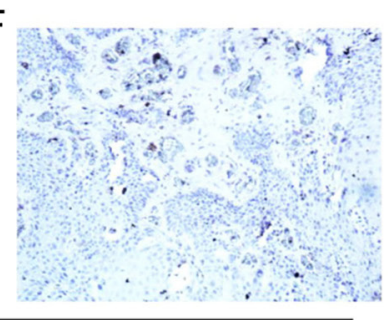

Hemorrhoids

G

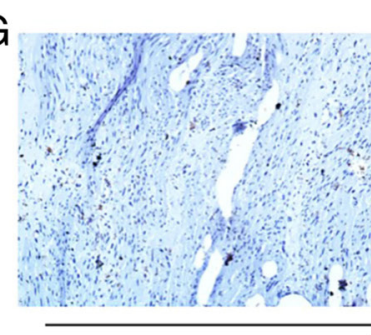

$\mathrm{H}$

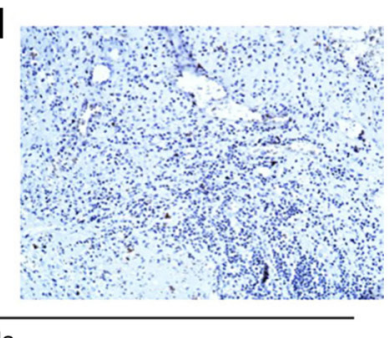

Figure 5. Influence of Anoac-H on the expression of RANTES and VEGF in patients with anorectal disease. (A and B) Expression of (A) RANTES and (B) VEGF in hemorrhoids. (C and D) Expression of (C) RANTES and (D) VEGF in fistula analyzed using IHC ( $\mathrm{n}=8$ ). (E and F) Expression of (E) RANTES and (F) VEGF in Anoac-H-treated hemorrhoids. (G and H) Expression of (G) RANTES and (H) VEGF in Anoac-H-treated fistula analyzed using IHC ( $\mathrm{n}=6$ ). All the images were captured using $\mathrm{x} 20$ objective. IHC, immunohistochemistry; RANTES, regulated upon activation, normal T cell expressed and presumably secreted.

treatment of post-hemorrhoidectomy wounds with triclosan solution is safe and it reduces the wound healing time compared to the control (40). Yet another study revealed that administration of mesoglycan prevented postoperative thrombosis and reduced pain after an open diathermy excisional hemorrhoidectomy surgery (41). Furthermore, non-surgical treatments such as topical and pharmacological approaches have not been fully effective. Treatment with Proctosoll Allevia ${ }^{\circledR}$ was proven effective against I-II degree symptomatic hemorrhoids with a good profile of tolerability and safety (42). A clinical study by our group suggested that treatment with Anoac-H polyherbal formulation exhibited efficacy and safety in patients with bleeding hemorrhoids (25). However, the mechanisms of its action on bleeding hemorrhoids and fistula have remained to be fully elucidated. Previous studies indicated that the treatment modalities that were demonstrated to alleviate inflammation achieved great clinical successes in the management of anorectal diseases (7). In particular, traditional medicine has proven effective for the clinical control of hemorrhoids and fistula by reducing inflammation $(24,43)$. In the present study, it was demonstrated that Anoac- $\mathrm{H}$, a traditional medicine, exerts its action on bleeding hemorrhoids, possibly by suppressing migration of immune and other cell types. The in vitro assays as well as clinical specimen analyses indicated that treatment with Anoac-H led to downregulation of RANTES and VEGF in cell lines as well as tissues. Anoac-H may reduce inflammation by downregulating RANTES and VEGF thereby controls hemorrhoids and fistula. VEGF is also known to be involved in angiogenesis as a proangiogenic factor. Anoac- $\mathrm{H}$ may reduce vascular density by downregulating VEGF in cell lines and clinical tissues, as vascular density drastically increases in these diseases (7). Anoac- $\mathrm{H}$ formulation consists of the extracts of Mimosa pudica, Euphorbia hirta, Messua ferrea and Berberis aristate. All of these traditional medicines were reported to exhibit anti-inflammatory activities in in vitro/in vivo models (44-47). The present results are consistent with previous studies that support the
A

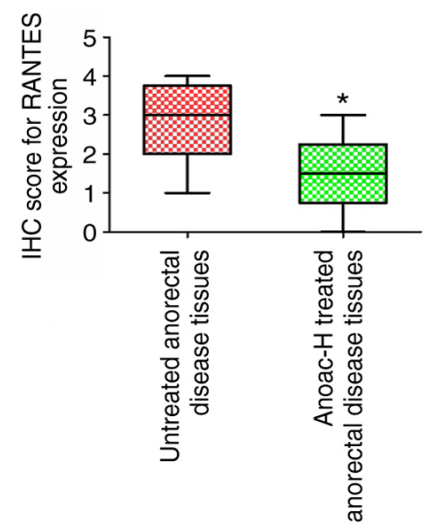

B

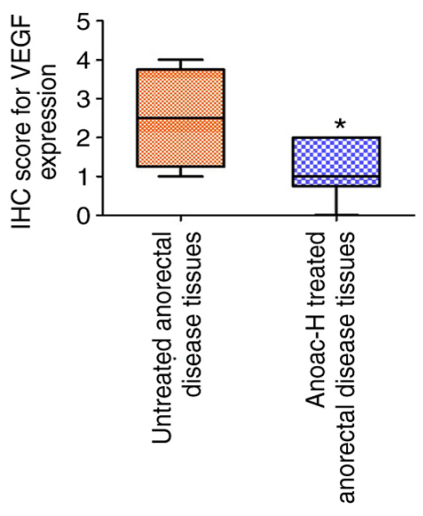

Figure 6. Influence of Anoac-H on IHC score of RANTES and VEGF in patients with anorectal disease. Box plots presenting the IHC score for (A) RANTES expression and (B) VEGF expression. ${ }^{*} \mathrm{P}<0.05$ vs. untreated group. IHC, immunohistochemistry; RANTES, regulated upon activation, normal $\mathrm{T}$ cell expressed and presumably secreted.

anti-inflammatory activity of these medicinal plants. An earlier clinical study by our group reported that $95 \%$ of the patients treated with Anoac-H had recovered from bleeding hemorrhoids. Furthermore, in the present study, it was demonstrated that the action of Anoac-H on bleeding hemorrhoids was due to its effect on migration, inflammation and downregulation of RANTES and VEGF. Although conclusive results may be obtained with a smaller number of patient samples, the number of anorectal samples is a major limitation of the present study. In the future, further experiments will be performed to validate the present results with a larger number of samples and the molecular mechanisms of the anti-inflammatory effects of Anoac-H will be comprehensively investigated.

In conclusion, the present study identified that Anoac-H does not affect the viability of fibroblasts and macrophages; however, it significantly decreases the migration of these cells. 
Furthermore, it suppressed the expression of RANTES and VEGF. It may be speculated that Anoac-H exerts its effects on hemorrhoids and fistula by alleviating inflammation and affecting the vasculature at the lesion sites. The present results highlight the implications of Anoac-H in the management of bleeding hemorrhoids and fistula.

\section{Acknowledgements}

Not applicable.

\section{Funding}

No funding was received.

\section{Availability of data and materials}

The datasets used and/or analyzed during the current study are available from the corresponding author on reasonable request.

\section{Authors' contributions}

Experiments were conceptually designed by AP, GCK, GB and RB. Herbal material was prepared by AP and GB. Experiments were performed and the manuscript was written by RB. AP, GCK and RB were conducted the analysis. The study was supervised by GCK. The manuscript was revised by AP, GCK and GB. AP and GCK confirmed the authenticity of all the raw data. All authors have read and approved the final manuscript.

\section{Ethics approval and consent to participate}

This study was approved by the Institutional Ethics Committee of Healing Hands Clinic (Pune, India). Written informed consent for participation in the study or use of their tissue was obtained from all participants.

\section{Patient consent for publication}

Not applicable.

\section{Competing interests}

The authors declare that they have no competing interests. GB and RB worked for the company Healing Hands \& Herbs.

\section{References}

1. Foxx-Orenstein AE, Umar SB and Crowell MD: Common anorectal disorders. Gastroenterol Hepatol (NY) 10: 294-301, 2014.

2. Guindic LC: Treatment of uncomplicated hemorrhoids with a Hemor-Rite ${ }^{\circledR}$ cryotherapy device: A randomized, prospective, comparative study. J Pain Res 7: 57-63, 2014.

3. Lorenzo-Rivero S: Hemorrhoids: Diagnosis and current management. Am Surg 75: 635-642, 2009.

4. Sun Z and Migaly J: Review of hemorrhoid disease: Presentation and management. Clin Colon Rectal Surg 29: 22-29, 2016.

5. Riss S, Weiser FA, Riss T, Schwameis K, Mittlböck M and Stift A: Haemorrhoids and quality of life. Colorectal Dis 13: e48-e52, 2011.
6. Peery AF, Sandler RS, Galanko JA, Bresalier RS, Figueiredo JC, Ahnen DJ, Barry EL and Baron JA: Risk factors for hemorrhoids on screening colonoscopy. PLoS One 10: e0139100, 2015.

7. Lohsiriwat V: Hemorrhoids: From basic pathophysiology to clinical management. World J Gastroenterol 18: 2009-2017, 2012.

8. Shrivastava L, da Silva Borges G and Shrivastava R: Clinical efficacy of a dual action, topical anti-edematous and antiinflammatory device for the treatment of external hemorrhoids. Clin Exp Pharmacol 8: 1-7, 2018.

9. Srivastava A, Yadav SK, Yachha SK, Thomas MA, Saraswat VA and Gupta RK: Pro-inflammatory cytokines are raised in extrahepatic portal venous obstruction, with minimal hepatic encephalopathy. J Gastroenterol Hepatol 26: 979-986, 2011.

10. Wojdasiewicz P, Poniatowski ŁA and Szukiewicz D: The role of inflammatory and anti-inflammatory cytokines in the pathogenesis of osteoarthritis. Mediators Inflamm 2014: 561459, 2014

11. Gulati K, Guhathakurta S, Joshi J, Rai N and Ray AJ: Cytokines and their role in health and disease: A brief overview. MOJ Immunol 4: 1-9, 2016.

12. Schall TJ: Biology of the RANTES/SIS cytokine family. Cytokine 3: 165-183, 1991.

13. Appay V, Brown A, Cribbes S, Randle E and Czaplewski LG: Aggregation of RANTES is responsible for its inflammatory properties. Characterization of nonaggregating, noninflammatory RANTES mutants. J Biol Chem 274: 27505-27512, 1999.

14. Nottingham JM and Rentea RM: Anal Fistulotomy (Seton Placement). StatPearls Publishing, Treasure Island, FL, 2020.

15. Gardner IH, Siddharthan RV and Tsikitis VL: Benign anorectal disease: Hemorrhoids, fissures, and fistulas. Ann Gastroenterol 33: 9-18, 2020.

16. Haddow JB, Musbahi O, MacDonald TT and Knowles CH: Comparison of cytokine and phosphoprotein profiles in idiopathic and Crohn's disease-related perianal fistula. World J Gastrointest Pathophysiol 10: 42-53, 2019.

17. Wang K, Deng P, Sun Y, Ye P, Zhang A, Wu C, Yue Z, Chen Z and Xia J: MicroRNA-155 promotes neointimal hyperplasia through smooth muscle-like cell-derived RANTES in arteriovenous fistulas. J Vasc Surg 67: 933-944.e3, 2018.

18. Sheikh P and Baakza A: Management of fistula-in-ano-the current evidence. Indian J Surg 76: 482-486, 2014.

19. Emile SH: Recurrent anal fistulas: When, why, and how to manage? World J Clin Cases 8: 1586-1591, 2020.

20. Azeemuddin M, Viswanatha GL, Rafiq M, Thippeswamy AH, Baig MR, Kavya KJ, Patki PS and Shyam R: An improved experimental model of hemorrhoids in rats: Evaluation of antihemorrhoidal activity of an herbal formulation. ISRN Pharmacol 2014: 530931, 2014.

21. Barnhoorn MC, Hakuno SK, Bruckner RS, Rogler G, Hawinkels LJAC and Scharl M: Stromal cells in the pathogenesis of inflammatory bowel disease. J Crohns Colitis 14: 995-1009, 2020.

22. van Onkelen RS, Gosselink MP, van Meurs M, Melief MJ, Schouten WR and Laman JD: Pro-inflammatory cytokines in cryptoglandular anal fistulas. Tech Coloproctol 20: 619-625, 2016.

23. Rakinic J and Poola VP: Hemorrhoids and fistulas: New solutions to old problems. Curr Probl Surg 51: 98-137, 2014.

24. Hashempur MH, Khademi F, Rahmanifard M and Zarshenas MM: An evidence-based study on medicinal plants for hemorrhoids in medieval Persia. J Evid Based Complementary Altern Med 22: 969-981, 2017.

25. Porwal A, Gandhi P and Kulkarni D: A prospective, open-label, single arm, single center study to evaluate safety and efficacy of ANOAC-H tablet in the treatment of bleeding hemorrhoids grades 1-3. Int J Ayurveda, 2018

26. Kumar D, Haldar S, Gorain M, Kumar S, Mulani FA, Yadav AS, Miele L, Thulasiram HV and Kundu GC: Epoxyazadiradione suppresses breast tumor growth through mitochondrial depolarization and caspase-dependent apoptosis by targeting PI3K/Akt pathway. BMC Cancer 18: 52, 2018.

27. Leopold JA, Walker J, Scribner AW, Voetsch B, Zhang YY, Loscalzo AJ, Stanton RC and Loscalzo J: Glucose-6-phosphate dehydrogenase modulates vascular endothelial growth factor-mediated angiogenesis. J Biol Chem 278: 32100-32106, 2003.

28. Butti R, Nimma R, Kundu G, Bulbule A, Kumar TV, Gunasekaran VP, Tomar D, Kumar D, Mane A, Gill SS, et al: Tumor-derived osteopontin drives the resident fibroblast to myofibroblast differentiation through Twist1 to promote breast cancer progression. Oncogene 40: 2002-2017, 2021. 
29. Kumar D, Kumar S, Gorain M, Tomar D, Patil HS, Radharani NN Kumar TV, Patil TV, Thulasiram HV and Kundu GC: Notch1-MAPK signaling axis regulates $\mathrm{CD} 133^{+}$cancer stem cell-mediated melanoma growth and angiogenesis. J Invest Dermatol 136: 2462-2474, 2016.

30. Tse GN: Practical management of hemorrhoids: Pitfalls and plain sailing. Can Fam Physician 34: 655-659, 1988.

31. Scharl M and Rogler G: Pathophysiology of fistula formation in Crohn's disease. World J Gastrointest Pathophysiol 5: 205-212, 2014.

32. Pata F, Sgró A, Ferrara F, Vigorita V, Gallo G and Pellino G: Anatomy, physiology and pathophysiology of haemorrhoids. Rev Recent Clin Trials 16: 75-80, 2021.

33. Mott T, Latimer K and Edwards C: Hemorrhoids: Diagnosis and treatment options. Am Fam Physician 97: 172-179, 2018.

34. Lohsiriwat V: Treatment of hemorrhoids: A coloproctologist's view. World J Gastroenterol 21: 9245-9252, 2015.

35. Moggia E, Talamo G, Gallo G, Bianco A, Barattini M, Salsano G, Zefiro D, Stefanini T and Berti S: Do we have another option to treat bleeding hemorrhoids? The Emborrhoid technique: Experience in 16 patients. Rev Recent Clin Trials 16: 81-86, 2021.

36. Lu M, Shi GY, Wang GQ, Wu Y, Liu Y and Wen H: Milligan-Morgan hemorrhoidectomy with anal cushion suspension and partial internal sphincter resection for circumferentia mixed hemorrhoids. World J Gastroenterol 19: 5011-5015, 2013.

37. Nienhuijs S and de Hingh I: Conventional versus LigaSure hemorrhoidectomy for patients with symptomatic Hemorrhoids Cochrane Database Syst Rev: Jan 21, 2009 (Epub ahead of print). doi: 10.1002/14651858.CD006761.pub2.

38. Patti R, Almasio PL, Muggeo VM, Buscemi S, Arcara M, Matranga S and Di Vita G: Improvement of wound healing after hemorrhoidectomy: A double-blind, randomized study of botulinum toxin injection. Dis Colon Rectum 48: 2173-2179, 2005.

39. Khan KI, Waqas A, Akmal M, Mahmood S and Iqbal A: Efficacy of combination of $0.2 \%$ GTN and lignocaine ointments in wound healing and pain relief after Milligan Morgan hemorrhoidectomy-a comparison with lignocaine and $0.2 \%$ GTN ointments separately. Int J Surg 12: 329-333, 2014.
40. Giannini I, Pecorella G, Pennisi D, Santangelo G, Digennaro R, Latorre F, Giuliani $G$ and Altomare DF: Control of post-hemorrhoidectomy symptoms and wound healing by Triclosan: A randomized, double-blind, controlled trial. Minerva Chir 69: 75-82, 2014.

41. Gallo G, Mistrangelo M, Passera R, Testa V, Pozzo M, Perinotti R, Lanati I, Lazzari I, Tonello P, Ugliono E, et al: Efficacy of mesoglycan in pain control after excisional hemorrhoidectomy: A pilot comparative prospective multicenter study. Gastroenterol Res Pract 2018: 6423895, 2018.

42. Sturiale A, Gallo G, Brusciano L, Cacace C, Cafaro D, Celedon Porzio F and Naldini G: Safety and efficacy of proctosoll allevia in the management of haemorrhoidal disease in adults: A prospective randomized clinical trial. Rev Recent Clin Trials 15: 152-159, 2020.

43. Derakhshan AR: Natural treatments for fissure in ano used by traditional Persian scholars, Razi (Rhazes) and Ibn Sina (Avicenna). J Evid Based Complementary Altern Med 22: 324-333, 2017.

44. MistryS,Patidar R, Vyas V,Jena J andDuttKR: Anti-inflammatory activity of Mimosa Pudica Linn. (Mimosaceae) leaves: An ethnpharmacological study. Int J Pharm Sci Res 4: 1789, 2012.

45. Xia M, Liu L, Qiu R, Li M, Huang W, Ren G and Zhang J: Anti-inflammatory and anxiolytic activities of Euphorbia hirta extract in neonatal asthmatic rats. AMB Express 8: 179, 2018.

46. Murthuza S and Manjunatha BK: In vitro and in vivo evaluation of anti-inflammatory potency of Mesua ferrea, Saraca asoca, Viscum album and Anthocephalus cadamba in murine macrophages raw 264.7 cell lines and wistar albino rats. Beni-Seuf Univ J Appl Sci 7: 719-723, 2018.

47. Nimisha, Rizvi DA, Fatima Z, Neema and Kaur CD: Antipsoriatic and anti-inflammatory studies of Berberis aristata extract loaded nanovesicular gels. Pharmacogn Mag 13 (Suppl 3): S587-S594, 2017.

This work is licensed under a Creative Commons Attribution-NonCommercial-NoDerivatives 4.0 International (CC BY-NC-ND 4.0) License. 